\title{
Adjunction between crossed modules of groups and algebras
}

\author{
J. M. Casas • N. Inassaridze • E. Khmaladze • \\ M. Ladra
}

Dedicated to Hvedri Inassaridze on the occasion of his 80th birthday

Received: 3 April 2013 / Accepted: 27 November 2013 / Published online: 8 January 2014

(C) Tbilisi Centre for Mathematical Sciences 2013

\begin{abstract}
We construct a pair of adjoint functors between the categories of crossed modules of groups and associative algebras and establish an equivalence of categories between module structures over a crossed module of groups and its respective crossed module of associative algebras.
\end{abstract}

Keywords Crossed module $\cdot$ Group $\cdot$ Associative algebra $\cdot$ Universal enveloping algebra Adjoint functors

Communicated by Ronald Brown.

The authors are indebted to the referee for his help in improving the presentation of this paper. They were supported by MICINN grant MTM 2009-14464-C02 (European FEDER support included). The second and the third authors were also supported by Shota Rustaveli National Science Foundation, grant DI/12/5-103/11 and Volkswagen Foundation, grant 85989. The third and the fourth authors were also supported by Xunta de Galicia, grants EM2013/016 and GRC2013-045 (European FEDER support included).

\section{J. M. Casas}

Departamento de Matemática Aplicada I, Universidad de Vigo,

E.E. Forestal, 36005 Pontevedra, Spain

e-mail: jmcasas@uvigo.es

N. Inassaridze $\cdot$ E. Khmaladze

A. Razmadze Mathematical Institute of I. Javakhishvili Tbilisi State University,

Tamarashvili Str. 6, 0177 Tbilisi, Georgia

e-mail: niko.inas@gmail.com

N. Inassaridze

Georgian Technical University, Tbilisi, Georgia

N. Inassaridze $\cdot$ E. Khmaladze

Tbilisi Centre for Mathematical Sciences, Tbilisi, Georgia 


\section{Introduction}

The general concept of a crossed module originates in the work of Whitehead in the late 1940s [17]. There the crossed modules were crossed modules of groups, introduced as algebraic models for path-connected CW-spaces whose homotopy groups are trivial in dimensions $>2$. Since their introduction, crossed modules have played an important role in homotopy theory. For illustration we mention various classification problems for low-dimensional homotopy types and the derivation of van Kampen theorem generalizations (see e.g. $[3,4]$ ).

Crossed modules of associative algebras are known at least as analogues of crossed modules of groups in another category. They have been investigated by various authors. In the works of Dedecker and Lue $[5,14]$ crossed modules of associative algebras have played a central role in what must be coefficients in low-dimensional non-abelian cohomology. In [1] Baues and Minian have shown that crossed modules of associative algebras can be used to represent the Hochschild cohomology. In the recent article [6], the Hochschild and (cotriple) cyclic homologies of crossed modules of associative algebras have been constructed and investigated.

The aim of the present paper is to give a relation between the categories of crossed modules of groups and (unital) associative algebras. Various definitions of modules over crossed modules of groups and associative algebras are examined. The classical fact that the group algebra functor is left adjoint to the unit group functor is extended to the categories of crossed modules, in such a way that the well-known equivalence of module structures is preserved. This result has already been noted and proved by Forrester-Barker [10], in his unpublished thesis, from a slightly different way.

Our constructions can be applied to develop (co)homology theories with coefficients for crossed modules of groups and associative algebras, and to extend the well-known Mac Lane isomorphism between the homology of a group and the Hochschild homology of its group algebra. It might be interesting to consider the main results here at the 2-category level and to relate our constructions with the representation theory of 2-groups developed by Elgueta [7]. Clearly, the investigation of possible Hopf algebra structure of our group algebra crossed module in the context of [9] and [11] is another prospective direction.

\section{Organisation}

After the introductory Sect. 1, the paper is organized in three sections. In Sect. 2, extending the definition of the actor of a crossed module of groups [15], we introduce

\footnotetext{
Present Address:

E. Khmaladze

Departamento de Matemática Aplicada I, Universidad de Vigo,

E.E. Forestal, 36005 Pontevedra, Spain

e-mail: e.khmal@gmail.com

\section{Ladra $(\varangle)$}

Departamento de Álgebra, Universidad de Santiago de Compostela, 15782 Santiago de Compostela, Spain

e-mail: manuel.ladra@usc.es
} 
the notion of module over a crossed module of groups over a ground ring $\mathbf{k}$ (Definition 2.2). In the equivalent cat ${ }^{1}$ setting, this notion is the same as a $\mathbf{k}$-linear representation of the corresponding cat ${ }^{1}$-group (Theorem 2.4) examined in [10]. In Sect. 3, we construct a crossed module of endomorphisms of an abelian crossed module of algebras, and a morphism to it is called a module structure over a crossed module of algebras (Definition 3.2). This definition is then translated to the equivalent cat ${ }^{1}$ setting (Definition 3.3, Proposition 3.5). In Sect. 4, we construct a functor $\mathbb{X U}: \mathrm{XUAlg} \rightarrow \mathrm{XGr}$, sending the crossed module of endomorphisms of algebras to the actor crossed module of groups (Lemma 4.3). Then we construct a left adjoint functor to $\mathbb{X} \mathbb{U}$ (Theorem 4.5). Consequently, we establish an equivalence of categories between the module structures over a crossed module of groups and over its respective crossed module of algebras (Theorem 4.6).

\section{Notations and conventions}

Throughout $\mathbf{k}$ is a commutative ring with unit. Algebras are (not necessarily unital) associative algebras over $\mathbf{k}$ and their category is denoted by Alg, while its subcategory of unital algebras is denoted by UAlg. We denote by Gr the category of groups.

\section{Crossed modules of groups}

\subsection{Basic notions}

A crossed module of groups $(H, G, \mu)$ is a group homomorphism $\mu: H \rightarrow G$ together with an action $(g, h) \mapsto^{g} h$ of $G$ on $H$ such that, for all $h, h^{\prime} \in H$ and $g \in G$,

$$
\mu\left({ }^{g} h\right)=g \mu(h) g^{-1}, \quad \mu(h) h^{\prime}=h h^{\prime} h^{-1} .
$$

A morphism of crossed modules of groups $(\varphi, \psi):(H, G, \mu) \rightarrow\left(H^{\prime}, G^{\prime}, \mu^{\prime}\right)$ is a pair of group homomorphisms $\left(\varphi: H \rightarrow H^{\prime}, \psi: G \rightarrow G^{\prime}\right)$ such that $\mu^{\prime} \varphi=\psi \mu$ and $\varphi\left({ }^{g} h\right)={ }^{\psi(g)} \varphi(h)$ for all $g \in G, h \in H$. We denote by XGr the category of crossed modules of groups. There are several categories equivalent to the category XGr. We mention the equivalences of crossed modules with simplicial groups whose Moore complexes are of length 1, with internal categories in the category of groups and with cat ${ }^{1}$-groups [13]. The equivalence between crossed modules of groups and cat ${ }^{1}$-groups will be used in what follows and we give it briefly immediately below.

A cat ${ }^{1}$-group $\mathfrak{C}=\left(G_{1}, G_{0}, s, t\right)$ consists of a group $G_{1}$ together with a subgroup $G_{0}$ and structural homomorphisms $s, t: G_{1} \rightarrow G_{0}$ satisfying

$$
\left.s\right|_{G_{0}}=\left.t\right|_{G_{0}}=\operatorname{id}_{G_{0}} \text { and }[\operatorname{Ker} s, \operatorname{Ker} t]=1 .
$$

A morphism of cat $^{1}{ }^{1}$ it -groups $\mathfrak{C} \rightarrow \mathfrak{C}^{\prime}$ is a group homomorphism $\gamma: G_{1} \rightarrow G_{1}^{\prime}$ such that $\left.\gamma\right|_{G_{0}} \subseteq G_{0}^{\prime}$ and $s^{\prime} \gamma=\left.\gamma\right|_{G_{0}} s, t^{\prime} \gamma=\left.\gamma\right|_{G_{0}} t$.

Given a crossed module of groups $(H, G, \mu)$, the corresponding cat ${ }^{1}$-group is $(H \rtimes G, G, s, t)$, where $s(h, g)=g, t(h, g)=\mu(h) g$ for all $(h, g) \in H \rtimes G$. 
Conversely, given a cat ${ }^{1}$-group $\left(G_{1}, G_{0}, s, t\right)$, the corresponding crossed module is $\left.t\right|_{\text {Ker } s}:$ Ker $s \rightarrow G_{0}$ with the action of $G_{0}$ on Ker $s$ given by conjugation.

\subsection{Actions of crossed modules of groups}

In [15], Norrie defined an action of a crossed module $(H, G, \mu)$ on another crossed module of groups $(M, P, \partial)$ as a crossed module morphism $(\varphi, \psi):(H, G, \mu) \rightarrow$ $\operatorname{Act}(M, P, \partial)$, where $\operatorname{Act}(M, P, \partial)=(\mathrm{D}(P, M)$, Aut $(\partial), \Delta)$ is the actor crossed module of $(M, P, \partial)$.

Let us recall briefly the construction of $\operatorname{Act}(M, P, \partial)$ from [15]. For the crossed module $(M, P, \partial)$, consider the set $\operatorname{Der}(P, M)$ of all derivations from $P$ to $M$, i.e. all maps $d: P \rightarrow M$ such that $d\left(p p^{\prime}\right)=d(p)^{p} d\left(p^{\prime}\right), p, p^{\prime} \in P$. Following Whitehead [17], there is a multiplication o in $\operatorname{Der}(P, M)$ given by

$$
\left(d_{1} \circ d_{2}\right)(p)=d_{1} \partial d_{2}(p) d_{2}(p) d_{1}(p), \quad p \in P,
$$

which turns $\operatorname{Der}(P, M)$ into a monoid. The Whitehead group $\mathrm{D}(P, M)$ is defined to be the group of units of $\operatorname{Der}(P, M)$. Further, Aut $(\partial)$ is the group of automorphisms of $(M, P, \partial)$ in the category XGr. The homomorphism $\Delta: \mathrm{D}(P, M) \rightarrow \operatorname{Aut}(\partial)$ is given by

$\Delta(d)=\left(\sigma_{d}, \theta_{d}\right)$, where $\sigma_{d}(m)=d \partial(m) m, \theta_{d}(p)=\partial d(p) p$, for $m \in M, p \in P$.

The action of $\operatorname{Aut}(\partial)$ on $\mathrm{D}(P, M)$ is defined by

$$
{ }^{(\sigma, \theta)} d=\sigma d \theta^{-1},(\sigma, \theta) \in \operatorname{Aut}(\partial), d \in \mathrm{D}(P, M) .
$$

There is an equivalent description of actions of crossed modules of groups.

Proposition 2.1 Let $(H, G, \mu)$ and $(M, P, \partial)$ be crossed modules of groups. Then there is an action of $(H, G, \mu)$ on $(M, P, \partial)$ if and only if the following conditions hold:

(i) The group $G$ (and so $H$ ) acts on $M$ and $P$, $\partial$ is a $G$-equivariant homomorphism, that is, $\partial\left({ }^{g} m\right)={ }^{g} \partial(m)$ and the action of $P$ on $M$ is a $G$-equivariant action (see e.g. [12, Definition 1]), that is, $\left.{ }^{g}\left({ }^{p} m\right)={ }^{(g} p\right)\left({ }^{g} m\right)$ for all $g \in G, m \in M, p \in P$;

(ii) There is a map $\xi: H \times P \rightarrow M$ such that

$$
\begin{aligned}
& \partial \xi(h, p)={ }^{\mu(h)} p p^{-1}, \\
& \xi(h, \partial(m))={ }^{\mu(h)} m m^{-1}, \\
& g_{\xi}(h, p)=\xi\left({ }^{g} h,{ }^{g} p\right), \\
& \xi\left(h h^{\prime}, p\right)={ }^{\mu(h)} \xi\left(h^{\prime}, p\right) \xi(h, p), \\
& \xi\left(h, p p^{\prime}\right)=\xi(h, p)^{p} \xi\left(h, p^{\prime}\right)
\end{aligned}
$$

for $h, h^{\prime} \in H, p, p^{\prime} \in P, m \in M, g \in G$. 
Proof Let $(H, G, \mu)$ act on $(M, P, \partial)$ by the following crossed module morphism

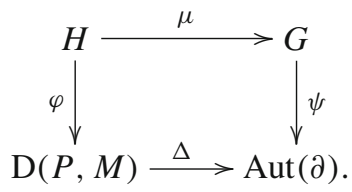

Clearly the homomorphism $\psi$ defines group actions of $G$ on $M$ and $P$ such that all statements in (i) hold. Define $\xi: H \times P \rightarrow M$ by $\xi(h, p)=\varphi(h)(p)$. Then the commutativity of the diagram (7) amounts precisely to the equalities (2) and (3); the equality (4) is equivalent to $\varphi\left({ }^{g} h\right)={ }^{\psi(g)} \varphi(h)$; whilst (5) and (6) are equivalent to the facts that $\varphi$ is a homomorphism and $\varphi(g)$, for any $g \in G$, is a derivation, respectively. The inverse statement is obvious.

\subsection{Modules over crossed modules of groups}

In [2], Beck introduced a convenient notion of coefficient module to be used in (co)homology theories. The notion makes sense in a broad context, and recovers the usual notions of module in familiar settings, such as groups, rings, commutative rings, Lie algebras, etc. Applying this general notion to the category of crossed modules of groups, one deduces that a module over a crossed module of groups $(H, G, \mu)$ is an abelian crossed module $(M, P, \partial)$ endowed with a $(H, G, \mu)$-action (see also [16]). It is known that an abelian crossed module of groups is just a homomorphism of abelian groups.

Now assume that $\partial: M \rightarrow P$ is a homomorphism of $\mathbf{k}$-modules. Let us denote by $\operatorname{Aut}_{\mathbf{k}}(\partial)$ the subgroup of $\operatorname{Aut}(\partial)$ of all k-automorphisms, and by $\mathrm{D}_{\mathbf{k}}(P, M)$ the subgroup of $\mathrm{D}(P, M)$ of all k-linear derivations whose inverse in $\mathrm{D}(P, M)$ is $\mathbf{k}$-linear as well. It is easy to check that the homomorphism

$$
\Delta_{\mathbf{k}}=\left.\Delta\right|_{\mathrm{D}_{\mathbf{k}}(P, M)}: \mathrm{D}_{\mathbf{k}}(P, M) \rightarrow \operatorname{Aut}_{\mathbf{k}}(\partial)
$$

is a crossed module of groups, denoted by $\operatorname{Act}_{\mathbf{k}}(M, P, \partial)$, where the action of $\operatorname{Aut}_{\mathbf{k}}(\partial)$ on $\mathrm{D}_{\mathbf{k}}(P, M)$ is induced by the action of $\operatorname{Aut}(\partial)$ on $\mathrm{D}(P, M)$.

Definition 2.2 Let $(H, G, \mu)$ be a crossed module of groups. A $(H, G, \mu)$-module over a commutative ring $\mathbf{k}$ is a homomorphism of $\mathbf{k}$-modules $\partial: M \rightarrow P$ together with a crossed module morphism $(\varphi, \psi):(H, G, \mu) \rightarrow \operatorname{Act}_{\mathbf{k}}(M, P, \partial)$.

Suppose $\partial: M \rightarrow P$ and $\partial^{\prime}: M^{\prime} \rightarrow P^{\prime}$ are $(H, G, \mu)$-modules over $\mathbf{k}$ with crossed module morphisms $(\varphi, \psi):(H, G, \mu) \rightarrow \operatorname{Act}_{\mathbf{k}}(M, P, \partial)$ and $\left(\varphi^{\prime}, \psi^{\prime}\right):(H, G, \mu) \rightarrow$ $\operatorname{Act}_{\mathbf{k}}\left(M^{\prime}, P^{\prime}, \partial^{\prime}\right)$, respectively. Then a morphism from $\partial: M \rightarrow P$ to $\partial^{\prime}: M^{\prime} \rightarrow P^{\prime}$ is a pair of homomorphisms of k-modules $\left(f_{M}: M \rightarrow M^{\prime}, f_{P}: P \rightarrow P^{\prime}\right)$ such that

$$
f_{P} \partial=\partial^{\prime} f_{M}, \quad\left(f_{M}, f_{P}\right) \psi(g)=\psi^{\prime}(g)\left(f_{M}, f_{P}\right), \quad f_{M} \varphi(h)=\varphi^{\prime}(h) f_{P}
$$

for all $g \in G$ and $h \in H$. There is an obvious composition of such morphisms and this leads to a definition of the category of $(H, G, \mu)$-modules over $\mathbf{k}$. 
Let us recall that Forrester-Barker in [10] developed the representation theory of cat ${ }^{1}$-groups. Namely, he constructed the automorphism cat ${ }^{1}$-group $\mathfrak{A u t}_{\mathbf{k}}(\partial)$ of a homomorphism of k-modules $\partial: M \rightarrow P$, and described a linear representation of a cat ${ }^{1}$-group $\mathfrak{C}$ as a morphism of cat $^{1}$-groups

$$
\phi: \mathfrak{C} \rightarrow \mathfrak{A} u t_{\mathbf{k}}(\partial)=\left(\operatorname{Homot}_{\mathbf{k}}(\partial), \operatorname{Aut}_{\mathbf{k}}(\partial), \mathfrak{s}, \mathfrak{t}\right)
$$

where $\operatorname{Homot}_{\mathbf{k}}(\partial)$ is the group of all homotopies between elements of $\operatorname{Aut}_{\mathbf{k}}(\partial)$ with the group structure defined as follows: if $h:\left(\sigma_{1}, \theta_{1}\right) \sim\left(\sigma_{2}, \theta_{2}\right)$ and $h^{\prime}:\left(\sigma_{1}^{\prime}, \theta_{1}^{\prime}\right) \sim\left(\sigma_{2}^{\prime}, \theta_{2}^{\prime}\right)$ are homotopies, then their product $h \# h^{\prime}$ is the homotopy $\sigma_{1} h^{\prime}+h \theta_{2}^{\prime}:\left(\sigma_{1} \sigma_{1}^{\prime}, \theta_{1} \theta_{1}^{\prime}\right) \sim$ $\left(\sigma_{2} \sigma_{2}^{\prime}, \theta_{2} \theta_{2}^{\prime}\right)$. Note that $\operatorname{Aut}_{\mathbf{k}}(\partial)$ is considered as a subgroup of $\operatorname{Homot}_{\mathbf{k}}(\partial)$ via the injection $\operatorname{Aut}_{\mathbf{k}}(\partial) \hookrightarrow \operatorname{Homot}_{\mathbf{k}}(\partial)$ sending each chain automorphism to the identity homotopy on it, while $\mathfrak{s}, \mathfrak{t}: \operatorname{Homot}_{\mathbf{k}}(\partial) \rightarrow \operatorname{Aut}_{\mathbf{k}}(\partial)$ are the homomorphisms selecting the source and the target of each homotopy.

We have the following

Lemma 2.3 Let $\partial: M \rightarrow P$ be a homomorphism of $\mathbf{k}$-modules. Then the $\mathrm{cat}^{1}{ }^{-}$ group $\left(\mathrm{D}_{\mathbf{k}}(P, M) \rtimes \operatorname{Aut}_{\mathbf{k}}(\partial), \operatorname{Aut}_{\mathbf{k}}(\partial), s, t\right)$ corresponding to the crossed module $\operatorname{Act}_{\mathbf{k}}(M, P, \partial)$ is isomorphic to $\mathfrak{A u t}_{\mathbf{k}}(\partial)$.

Proof It is routine to check that there are homomorphisms of groups

$$
\mathrm{D}_{\mathbf{k}}(P, M) \rtimes \operatorname{Aut}_{\mathbf{k}}(\partial) \underset{\beta}{\stackrel{\alpha}{\rightleftarrows}} \operatorname{Homot}_{\mathbf{k}}(\partial)
$$

defined, for all $(d,(\sigma, \theta)) \in \mathrm{D}_{\mathbf{k}}(P, M) \rtimes \operatorname{Aut}_{\mathbf{k}}(\partial)$ and $\left(h:\left(\sigma_{1}, \theta_{1}\right) \sim\left(\sigma_{2}, \theta_{2}\right)\right) \in$ $\operatorname{Homot}_{\mathbf{k}}(\partial)$, by the formulas

$$
\begin{aligned}
& \alpha(d,(\sigma, \theta))=\left(-d \theta:(\sigma, \theta) \sim\left(\sigma_{\delta} \sigma, \theta_{\delta} \theta\right)\right), \\
& \beta\left(h:\left(\sigma_{1}, \theta_{1}\right) \sim\left(\sigma_{2}, \theta_{2}\right)\right)=\left(-h \theta_{1}^{-1},\left(\sigma_{1}, \theta_{1}\right)\right) .
\end{aligned}
$$

Here we only note that $-h \theta_{1}^{-1} \in \mathrm{D}_{\mathbf{k}}(P, M)$, since it has an inverse $\sigma_{1} \sigma_{2}^{-1} h \theta_{1}^{-1}$. Clearly $\alpha$ and $\beta$ are inverses to each other, and they commute with the structural homomorphisms of the cat ${ }^{1}$-groups $\left(\mathrm{D}_{\mathbf{k}}(P, M) \rtimes \operatorname{Aut}_{\mathbf{k}}(\partial), \mathrm{Aut}_{\mathbf{k}}(\partial), s, t\right)$ and $\mathfrak{A u t}_{\mathbf{k}}(\partial)$.

Theorem 2.4 Let $(H, G, \mu)$ be a crossed module of groups. Then the category of $(H, G, \mu)$-modules over $\mathbf{k}$ is equivalent to the category of linear representations of the corresponding cat $^{1}$-group $(H \rtimes G, G, s, t)$.

Proof Straightforward from Lemma 2.3.

\section{Crossed modules of algebras}

\subsection{Basic notions}

We begin by recalling some basic notions about crossed modules of algebras from [6] (also cf. [8]). Let $A$ and $R$ be two algebras. By an action of $A$ on $R$ we mean an $A$-bimodule structure on $R$ satisfying the following conditions: 


$$
a\left(r r^{\prime}\right)=(a r) r^{\prime}, \quad(r a) r^{\prime}=r\left(a r^{\prime}\right), \quad\left(r r^{\prime}\right) a=r\left(r^{\prime} a\right)
$$

for all $a \in A, r, r^{\prime} \in R$. For example, if $A$ is a subalgebra of some algebra $B$ and $R$ is an ideal in $B$, then multiplication in $B$ yields an action of $A$ on $R$.

A crossed module of algebras $(R, A, \rho)$ is an algebra homomorphism $\rho: R \rightarrow A$ together with an action of $A$ on $R$ such that the following conditions hold:

$$
\begin{aligned}
& \rho(a r)=a \rho(r), \quad \rho(r a)=\rho(r) a, \\
& \rho(r) r^{\prime}=r r^{\prime}=r \rho\left(r^{\prime}\right)
\end{aligned}
$$

for all $a \in A, r, r^{\prime} \in R$. We point out that the image of $\rho$ is necessarily an ideal of $A$, and that $\operatorname{Ker}_{\rho}$, contained in the two-sided annihilator of $R$, is an $A / \rho R$-bimodule.

A morphism $(\alpha, \beta):(R, A, \rho) \rightarrow\left(R^{\prime}, A^{\prime}, \rho^{\prime}\right)$ of crossed modules is a pair of algebra homomorphisms $\left(\alpha: R \rightarrow R^{\prime}, \beta: A \rightarrow A^{\prime}\right)$ such that $\rho^{\prime} \alpha=\beta \rho, \alpha(a r)=$ $\beta(a) \alpha(r)$ and $\alpha(r a)=\alpha(r) \beta(a)$ for $a \in A, r \in R$. Let us denote the category of crossed modules of algebras by XAlg. Moreover, denote by XUAlg the subcategory of XAlg of crossed modules of unital algebras, whose objects are crossed modules $(R, A, \rho)$ with $A$ a unital algebra and for $A$-bimodule structure $1 r=r 1=r, r \in R$, while morphisms are crossed module morphisms $(\alpha, \beta)$ with $\beta$ a homomorphism of unital algebras.

A cat ${ }^{1}$-algebra $\left(A_{1}, A_{0}, \sigma, \tau\right)$ consists of an algebra $A_{1}$ together with a subalgebra $A_{0}$ and structural homomorphisms $\sigma, \tau: A_{1} \rightarrow A_{0}$ satisfying

$$
\left.\sigma\right|_{A_{0}}=\left.\tau\right|_{A_{0}}=\operatorname{id}_{A_{0}} \text { and } \operatorname{Ker} \sigma \operatorname{Ker} \tau+\operatorname{Ker} \tau \operatorname{Ker} \sigma=0
$$

Just as in the case of groups, a crossed module of algebras is equivalent to a cat ${ }^{1}$ algebra [8]. More precisely, given a crossed module of algebras $(R, A, \rho)$, the corresponding cat ${ }^{1}$-algebra is $(R \rtimes A, A, \sigma, \tau)$, where $R \rtimes A$ denotes the semi-direct product algebra with the underlying $\mathbf{k}$-module $R \oplus A$ endowed with the multiplication

$$
(r, a)\left(r^{\prime}, a^{\prime}\right)=\left(r r^{\prime}+a r^{\prime}+r a^{\prime}, a a^{\prime}\right)
$$

$\sigma(r, a)=a, \tau(r, a)=\rho(r)+a$ for all $(r, a), \quad\left(r^{\prime}, a^{\prime}\right) \in R \rtimes A$. Conversely, for a cat ${ }^{1}-$ algebra $\left(A_{1}, A_{0}, \sigma, \tau\right)$ the corresponding crossed module is $\left.\tau\right|_{\operatorname{Ker} \sigma}: \operatorname{Ker} \sigma \rightarrow A_{0}$ with the action of $A_{0}$ on $\operatorname{Ker} \sigma$ defined by the multiplication in $A_{1}$.

We need to remark that, under the aforementioned equivalence, objects of XUAlg correspond to that of unital cat ${ }^{1}$-algebras whose structural morphisms are homomorphisms of unital algebras.

\subsection{Modules over crossed modules of algebras}

In this subsection, for a given abelian crossed module of algebras, we construct a crossed module of endomorphisms and define a module structure over a crossed module of algebras as its morphism to the crossed module of endomorphisms. 
Given an abelian crossed module of algebras, that is, just a homomorphism of k-modules $\partial: V \rightarrow W$. Then the k-module $\operatorname{Hom}_{\mathbf{k}}(W, V)$ is an algebra with the multiplication defined by

$$
d_{1} * d_{2}=d_{1} \partial d_{2}
$$

for all $d_{1}, d_{2} \in \operatorname{Hom}_{\mathbf{k}}(W, V)$. Let $\operatorname{End}_{\mathbf{k}}(V, W, \partial)$ denote the algebra

$$
\left\{(\phi, \psi) \mid \phi \in \operatorname{End}_{\mathbf{k}}(V), \psi \in \operatorname{End}_{\mathbf{k}}(W), \psi \partial=\partial \phi\right\}
$$

The following lemma is straightforward from direct calculations.

\section{Lemma 3.1 The algebra homomorphism}

$$
\Theta: \operatorname{Hom}_{\mathbf{k}}(W, V) \rightarrow \operatorname{End}_{\mathbf{k}}(V, W, \partial), \quad d \mapsto(d \partial, \partial d),
$$

together with an algebra action of $\operatorname{End}_{\mathbf{k}}(V, W, \partial)$ on $\operatorname{Hom}_{\mathbf{k}}(W, V)$ given by

$(\phi, \psi) d=\phi d$ and $d(\phi, \psi)=d \psi, \quad d \in \operatorname{Hom}_{\mathbf{k}}(W, V),(\phi, \psi) \in \operatorname{End}_{\mathbf{k}}(V, W, \partial)$,

is a crossed module of unital algebras.

Definition 3.2 Let $(R, A, \rho)$ be a crossed module of algebras. A left $(R, A, \rho)$-module is an abelian crossed module of algebras $(V, W, \partial)$ together with a morphism of crossed modules of algebras $(R, A, \rho) \rightarrow\left(\operatorname{Hom}_{\mathbf{k}}(W, V), \operatorname{End}_{\mathbf{k}}(V, W, \partial), \Theta\right)$.

Suppose $(V, W, \partial)$ and $\left(V^{\prime}, W^{\prime}, \partial^{\prime}\right)$ are $(R, A, \rho)$-modules with morphisms of crossed modules of algebras $(\alpha, \beta):(R, A, \rho) \rightarrow\left(\operatorname{Hom}_{\mathbf{k}}(W, V), \operatorname{End}_{\mathbf{k}}(V, W, \partial), \Theta\right)$ and $\left(\alpha^{\prime}, \beta^{\prime}\right):(R, A, \rho) \rightarrow\left(\operatorname{Hom}_{\mathbf{k}}\left(W^{\prime}, V^{\prime}\right), \operatorname{End}_{\mathbf{k}}\left(V^{\prime}, W^{\prime}, \partial^{\prime}\right), \Theta^{\prime}\right)$, respectively. Then a morphism from $(V, W, \partial)$ to $\left(V^{\prime}, W^{\prime}, \partial^{\prime}\right)$ is a pair of homomorphisms of k-modules $\left(f_{V}: V \rightarrow V^{\prime}, f_{W}: W \rightarrow W^{\prime}\right)$ such that

$$
f_{W} \partial=\partial^{\prime} f_{V}, \quad\left(f_{V}, f_{W}\right) \beta(a)=\beta^{\prime}(a)\left(f_{V}, f_{W}\right), \quad f_{V} \alpha(r)=\alpha^{\prime}(r) f_{W}
$$

for all $a \in A$ and $r \in R$. There is an obvious composition of such morphisms and this leads to the definition of the category of $(R, A, \rho)$-modules.

Since crossed modules of algebras and cat ${ }^{1}$-algebras are equivalent, and we have a definition of left modules over a crossed module of algebras, this may also be considered as a left module of the corresponding cat ${ }^{1}$-algebra. However, a direct definition of a left module over a cat ${ }^{1}$-algebra will be also useful and we give it immediately below.

First, we need to recall from [10] that a cat ${ }^{1}$-module $\left(V_{1}, V_{0}, i, j\right)$ consists of a k-module $V_{1}$, a k-submodule $V_{0}$ of $V_{1}$ and structural morphisms $i, j: V_{1} \rightarrow V_{0}$ satisfying $\left.i\right|_{V_{0}}=\left.j\right|_{V_{0}}=\operatorname{id}_{V_{0}}$. 
Definition 3.3 A left module over a cat ${ }^{1}$-algebra $\left(A_{1}, A_{0}, \sigma, \tau\right)$ is a cat ${ }^{1}$-module $\left(V_{1}, V_{0}, i, j\right)$ together with a left action of $A_{1}$ on $V_{1}$ and a left action of $A_{0}$ on $V_{0}$ such that the structural morphisms commute with the actions, that is, $i\left(a_{1} v_{1}\right)=\sigma\left(a_{1}\right) i\left(v_{1}\right)$, $j\left(a_{1} v_{1}\right)=\tau\left(a_{1}\right) j\left(v_{1}\right)$ for all $a_{1} \in A_{1}, v_{1} \in V_{1}$ and the condition

$$
\operatorname{Ker} \sigma \operatorname{Ker} i+\operatorname{Ker} \tau \operatorname{Ker} j=0
$$

holds.

Remark 3.4 Forrester-Barker in [10] gave a definition of a left module over a cat ${ }^{1}$ algebra, but omitted the important condition (10). This condition is essential for proving equivalence to a left module over the corresponding crossed module of algebras and consequently the equivalence between modules over crossed modules of groups and the corresponding crossed modules of algebras (see our main result Theorem 4.6)

Proposition 3.5 Let $(R, A, \rho)$ be a crossed module of algebras. An abelian crossed module of algebras $(V, W, \partial)$ is a left module over $(R, A, \rho)$ if and only if the corresponding cat $^{1}$-module $(V \oplus W, W, i, j)$ is a left module over the corresponding cat $^{1}$-algebra $(R \rtimes A, A, \sigma, \tau)$.

Proof Let $(V, W, \partial)$ be a $(R, A, \rho)$-module. Then there is a morphism $(\alpha, \beta)$ of crossed modules of algebras

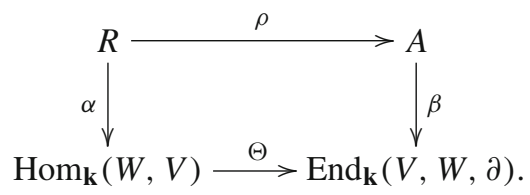

Suppose that $\beta$ has components $\beta_{1}: A \rightarrow \operatorname{End}_{\mathbf{k}}(V)$ and $\beta_{2}: A \rightarrow \operatorname{End}_{\mathbf{k}}(W)$, that is, $\beta(a)=\left(\beta_{1}(a), \beta_{2}(a)\right)$ for all $a \in A$. It is clear that $A$ acts (to the left) on $V$ and on $W: a v=\beta_{1}(a)(v)$ and $a w=\beta_{2}(a)(w)$ for all $a \in A, v \in V, w \in W$. It is routine to check that the equality

$$
(r, a)(v, w)=(\alpha(r)(w)+(\rho(r)+a) v, a w)
$$

defines a left action of the algebra $R \rtimes A$ on the k-module $V \oplus W$, which commutes with the structural morphisms.

In order to check condition (10), note that $\operatorname{Ker} \sigma$ (resp. $\operatorname{Ker} \tau, \operatorname{Ker} i, \operatorname{Ker} j$ ) consists of all elements of the form $(r, 0)$ (resp. $(r,-\rho(r)),(v, 0),(v,-\partial(v)))$ for $r \in R$, $v \in V$, and we have

$$
\begin{aligned}
& (r,-\rho(r))(v, 0)=(\alpha(r)(0)+(\rho(r)-\rho(r)) v, 0)=(0,0), \\
& (r, 0)(v,-\partial(v))=(-\alpha(r) \partial(v)+\rho(r) v, 0)=(0,0)
\end{aligned}
$$

since $\alpha(r) \partial(v)=\rho(r) v$ by commutativity of the diagram (11).

Conversely, given a left $(R \rtimes A, A, \sigma, \tau)$-module structure on the cat ${ }^{1}$-module ( $V \oplus W, W, i, j)$, we define the maps $\alpha$ and $\beta=\left(\beta_{1}, \beta_{2}\right)$ in the diagram (11) by the equalities 


$$
\begin{aligned}
(\alpha(r)(w), 0) & =(r, 0)(0, w), \\
\left(\beta_{1}(a)(v), 0\right) & =(0, a)(v, 0), \\
\beta_{2}(a)(w) & =a w
\end{aligned}
$$

for all $r \in R, a \in A, v \in V$ and $w \in W$. Now the remaining details are straightforward calculations and are left to the reader.

\section{Adjunction between XGr and XUAlg}

Given a unital algebra $A$, its subset of all invertible elements forms a group $\mathbb{U}(A)$ called the group of units of the algebra $A$. This gives a functor $\mathbb{U}:$ UAlg $\rightarrow$ Gr called unit group functor. Let $\mathbb{K}: \mathrm{Gr} \rightarrow$ UAlg denote its left adjoint functor, sending a group $G$ to its group algebra $\mathbb{K}(G)$, called the group algebra functor. In this section we construct adjoint functors $\mathbb{X U}:$ XUAlg $\rightarrow$ XGr and $\mathbb{X} \mathbb{K}:$ XGr $\rightarrow$ XUAlg, which are natural generalizations of the functors $\mathbb{U}$ and $\mathbb{K}$.

\subsection{The functor $\mathbb{X} \mathbb{U}$}

Given an object $(R, A, \rho)$ of XUAlg, consider its corresponding (unital) cat ${ }^{1}$-algebra

$$
R \rtimes A \underset{\tau}{\stackrel{\sigma}{\longrightarrow}} A .
$$

Applying the functor $\mathbb{U}$, we obtain a diagram of groups

$$
\mathbb{U}(R \rtimes A) \underset{\mathbb{U}(\tau)}{\stackrel{\mathbb{U}(\sigma)}{\rightrightarrows}} \mathbb{U}(A) .
$$

Lemma 4.1 (12) is a cat $^{1}$-group.

Proof It is clear that $\left.\mathbb{U}(\sigma)\right|_{\mathbb{U}(A)}=\left.\mathbb{U}(\tau)\right|_{\mathbb{U}(A)}=\mathrm{id} \mathbb{U}_{(A)}$. We only have to check that $[\operatorname{Ker} \mathbb{U}(\sigma), \operatorname{Ker} \mathbb{U}(\tau)]=1$.

For this we note that any element of $\operatorname{Ker} \mathbb{U}(\sigma)$ has the form $\left(r_{0}, 1\right)$, with $r_{0} \in R$ such that there exists $r_{0}^{\prime} \in R$ satisfying $r_{0} r_{0}^{\prime}+r_{0}^{\prime}+r_{0}=r_{0}^{\prime} r_{0}+r_{0}^{\prime}+r_{0}=0$. Moreover, any element of $\operatorname{Ker} \mathbb{U}(\tau)$ has the form $\left(r_{1}, 1-\rho\left(r_{1}\right)\right), r_{1} \in R$. Then we have

$$
\left(r_{0}, 1\right)\left(r_{1}, 1-\rho\left(r_{1}\right)\right)=\left(r_{0}+r_{1}, 1-\rho\left(r_{1}\right)\right)=\left(r_{1}, 1-\rho\left(r_{1}\right)\right)\left(r_{0}, 1\right) .
$$

This means that $\left[\left(r_{0}, 1\right),\left(r_{1}, 1-\rho\left(r_{1}\right)\right)\right]=1$ and the assertion follows.

Now we define the functor $\mathbb{X} U: X U A l g \rightarrow$ XGr by the formula

$$
\mathbb{X} \mathbb{U}(R, A, \rho)=\left(\operatorname{Ker} \mathbb{U}(\sigma), \mathbb{U}(A),\left.\mathbb{U}(\tau)\right|_{\operatorname{Ker} \mathbb{U}(\sigma)}\right), \quad(R, A, \rho) \in \mathrm{XUAlg} .
$$


The functor $\mathbb{X} \mathbb{U}$ is a natural generalization of the unit group functor $\mathbb{U}$ in the following sense: there are two ways of regarding a unital algebra $A$ (resp. a group $G$ ) as a crossed module of unital algebras (resp. crossed module of groups), via the trivial map 0:0 $\rightarrow A$ (resp. 1: $1 \rightarrow G$ ) and via the identity map $\operatorname{id}_{A}: A \rightarrow A$ (resp. $\operatorname{id}_{G}: G \rightarrow G$ ) with action of $A$ (resp. of $G$ ) on itself by multiplication (resp. by conjugation). There are full embeddings

$$
\mathbb{E}_{0}, \mathbb{E}_{1}: \mathrm{UAlg} \longrightarrow \mathrm{XUAlg} \quad \text { (resp. } \mathbb{E}_{0}^{\prime}, \mathbb{E}_{1}^{\prime}: \mathrm{Gr} \longrightarrow \mathrm{XGr} \text { ) }
$$

defined by $\mathbb{E}_{0}(A)=(0, A, 0), \mathbb{E}_{1}(A)=\left(A, A, \mathrm{id}_{A}\right)\left(\right.$ resp. $\mathbb{E}_{0}^{\prime}(G)=(1, G, 1)$, $\left.\mathbb{E}^{\prime}{ }_{1}(G)=\left(G, G, \operatorname{id}_{G}\right)\right)$. Then, it is easy to see that the following diagram is commutative

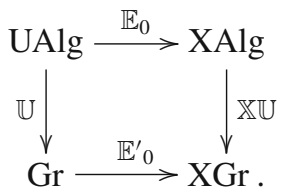

Moreover, we have the following

Proposition 4.2 There is a natural isomorphism of functors

$$
\mathbb{X U} \circ \mathbb{E}_{1} \cong \mathbb{E}_{1}^{\prime} \circ \mathbb{U}
$$

Proof Given a unital algebra $A$, by definition we have that $\mathbb{E}^{\prime}{ }_{1} \circ \mathbb{U}(A)=$ $\left(\mathbb{U}(A), \mathbb{U}(A), \operatorname{id}_{\mathbb{U}(A)}\right)$. Thus, we need to show that the crossed module of groups $\mathbb{X} \mathbb{U}\left(A, A, \mathrm{id}_{A}\right)\left(=\mathbb{X} \mathbb{U} \circ \mathbb{E}_{1}(A)\right)$ is isomorphic to $\left(\mathbb{U}(A), \mathbb{U}(A), \operatorname{id}_{\mathbb{U}(A)}\right)$. We follow the definition of $\mathbb{X} U$ and consider the cat ${ }^{1}$-algebra $(A \rtimes A, A, \sigma, \tau)$ with $\sigma\left(a, a^{\prime}\right)=a^{\prime}$, $\tau\left(a, a^{\prime}\right)=a+a^{\prime}$. Applying the functor $\mathbb{U}$ we arrive to the cat ${ }^{1}$-group

$$
\mathbb{U}(A \rtimes A) \underset{\mathbb{U}(\tau)}{\stackrel{\mathbb{U}(\sigma)}{\lessgtr}} \mathbb{U}(A) .
$$

We claim that $\left.\mathbb{U}(\tau)\right|_{\operatorname{Ker} \mathbb{U}(\sigma)}: \operatorname{Ker} \mathbb{U}(\sigma) \rightarrow \mathbb{U}(A)$ is an isomorphism of groups. Indeed, the assignment $u \mapsto(u-1,1)$, for any element $u \in \mathbb{U}(A)$, defines a homomorphism from $\mathbb{U}(A)$ to $\operatorname{Ker} \mathbb{U}(\sigma)$ which is a two-sided inverse for $\left.\mathbb{U}(\tau)\right|_{\operatorname{Ker} \mathbb{U}(\sigma)}$.

Now it is easy to see that the pair $\left(\left.\mathbb{U}(\tau)\right|_{\operatorname{Ker} \mathbb{U}(\sigma)}, \operatorname{id}_{\mathbb{U}(A)}\right)$ is an isomorphism between crossed modules of groups $\left(\operatorname{Ker} \mathbb{U}(\sigma), \mathbb{U}(A),\left.\mathbb{U}(\tau)\right|_{\operatorname{Ker} \mathbb{U}(\sigma)}\right)$ and $\left(\mathbb{U}(A), \mathbb{U}(A), \operatorname{id}_{\mathbb{U}(A)}\right)$, which provides the required isomorphism between the functors $\mathbb{X U} \circ \mathbb{E}_{1}$ and $\mathbb{E}_{1}^{\prime} \circ \mathbb{U}$.

Lemma 4.3 Let $\partial: V \rightarrow W$ be a $\mathbf{k}$-homomorphism. Then the crossed module of groups $\mathbb{X} \mathbb{U}\left(\operatorname{Hom}_{\mathbf{k}}(W, V), \operatorname{End}_{\mathbf{k}}(V, W, \partial), \Theta\right)$ is isomorphic to the actor crossed module $\operatorname{Act}_{\mathbf{k}}(V, W, \partial)=\left(\mathrm{D}_{\mathbf{k}}(W, V), \operatorname{Aut}_{\mathbf{k}}(\partial), \Delta_{\mathbf{k}}\right)$. 
Proof We follow the definition of $\mathbb{X} \mathbb{U}\left(\operatorname{Hom}_{\mathbf{k}}(W, V), \operatorname{End}_{\mathbf{k}}(V, W, \partial), \Theta\right)$ and consider the cat ${ }^{1}$-algebra

$$
\operatorname{Hom}_{\mathbf{k}}(W, V) \rtimes \operatorname{End}_{\mathbf{k}}(V, W, \partial) \stackrel{\sigma}{\underset{\tau}{\rightleftharpoons}} \operatorname{End}_{\mathbf{k}}(V, W, \partial)
$$

with $\sigma(d,(\phi, \psi))=(\phi, \psi)$ and $\tau(d,(\phi, \psi))=\Theta(d)+(\phi, \psi)=(d \partial+\phi, \partial d+\psi)$. It is clear that $\mathbb{U}\left(\operatorname{End}_{\mathbf{k}}(V, W, \partial)\right)=\operatorname{Aut}_{\mathbf{k}}(\partial)$. Further, an element $(d,(\phi, \psi)) \in$ $\operatorname{Hom}_{\mathbf{k}}(W, V) \rtimes \operatorname{End}_{\mathbf{k}}(V, W, \partial)$ is invertible if and only if $(\phi, \psi) \in \operatorname{Aut}_{\mathbf{k}}(\partial)$ and there exists another $d^{\prime} \in \operatorname{Hom}_{\mathbf{k}}(W, V)$ such that

$$
d \partial d^{\prime}+\phi d^{\prime}+d \psi^{-1}=0=d^{\prime} \partial d+\phi^{-1} d+d^{\prime} \psi
$$

It follows that $\operatorname{Ker} \mathbb{U}(\sigma)$ consists exactly of those $d \in \operatorname{Hom}_{\mathbf{k}}(W, V)=\operatorname{Der}_{\mathbf{k}}(W, V)$ which are invertible with respect to the product (1), that is, $\operatorname{Ker} \mathbb{U}(\sigma) \cong \mathrm{D}_{\mathbf{k}}(W, V)$. At the same time, for any $d \in \operatorname{Ker} \mathbb{U}(\sigma)$, we have

$$
\mathbb{U}(\tau)(d)=(d \partial, \partial d)+\left(\operatorname{id}_{V}, \mathrm{id}_{W}\right)=\left(d \partial+\mathrm{id}_{V}, \partial d+\mathrm{id}_{W}\right)=\Delta_{\mathbf{k}}(d) .
$$

This completes the proof.

\subsection{The functor $\mathbb{X} \mathbb{K}$}

Now we construct a left adjoint functor of the functor $\mathbb{X} \mathbb{U}$ (cf. Forrester-Barker's thesis, [10], where an equivalent construction is given).

Given a crossed module of groups $\mu: H \rightarrow G$ consider its corresponding cat ${ }^{1}$ -

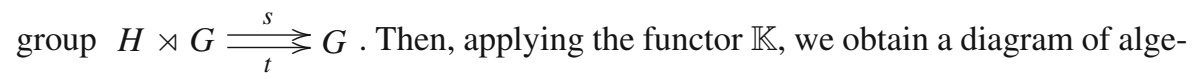
bras

$$
\mathbb{K}(H \rtimes G) \underset{\mathbb{K}(t)}{\stackrel{\mathbb{K}(s)}{\rightrightarrows}} \mathbb{K}(G) .
$$

It is easy to see that $\left.\mathbb{K}(s)\right|_{\mathbb{K}(G)}=\left.\mathbb{K}(t)\right|_{\mathbb{K}(G)}=\mathrm{id}_{\mathbb{K}(G)}$, but the other condition of $\mathrm{cat}^{1}$-algebra is not fulfilled in general. Thus consider a new diagram

$$
\mathbb{K}(H \rtimes G) / X \underset{\overline{\mathbb{K}}(t)}{\stackrel{\overline{\mathbb{K}}(s)}{\gtrless}} \mathbb{K}(G),
$$

where $X=\operatorname{Ker} \mathbb{K}(s) \operatorname{Ker} \mathbb{K}(t)+\operatorname{Ker} \mathbb{K}(t) \operatorname{Ker} \mathbb{K}(s), \overline{\mathbb{K}}(s)$ and $\overline{\mathbb{K}}(t)$ are induced by $\mathbb{K}(s)$ and $\mathbb{K}(t)$, respectively. What we have obtained is obviously a cat ${ }^{1}$-algebra. Define $\mathbb{X} \mathbb{K}(H, G, \mu)$ as the crossed module of algebras corresponding to the cat ${ }^{1}$-algebra (13), that is,

$$
\mathbb{X} \mathbb{K}(H, G, \mu)=\left(\operatorname{Ker} \overline{\mathbb{K}}(s), \mathbb{K}(G),\left.\overline{\mathbb{K}}(t)\right|_{\operatorname{Ker} \overline{\mathbb{K}}(s)}\right)
$$


It is easy to see that this construction determines a functor $\mathbb{X} \mathbb{K}: \mathrm{XGr} \rightarrow \mathrm{XUAlg}$, which is a natural generalization of the functor $\mathbb{K}$ in the sense of the following commutative diagram

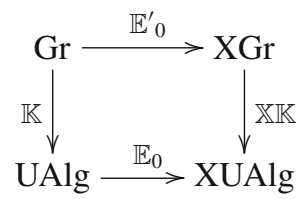

and the following proposition.

Proposition 4.4 There is a natural isomorphism of functors

$$
\mathbb{X} \mathbb{K} \circ \mathbb{E}_{1}^{\prime} \cong \mathbb{E}_{1} \circ \mathbb{K}
$$

Proof Given a group $G$, we have that $\mathbb{E}_{1} \circ \mathbb{K}(G)=\left(\mathbb{K}(G), \mathbb{K}(G), \operatorname{id}_{\mathbb{K}(G)}\right)$. Thus, we need to show that the crossed module of unital algebras $\mathbb{X} \mathbb{K}\left(G, G, \operatorname{id}_{G}\right)$ (= $\left.\mathbb{X} \mathbb{K} \circ \mathbb{E}_{1}^{\prime}(G)\right)$ is isomorphic to $\left(\mathbb{K}(G), \mathbb{K}(G), \mathrm{id}_{\mathbb{K}(G)}\right)$. We follow the definition of $\mathbb{X} \mathbb{K}$ and consider the cat ${ }^{1}$-group $(G \rtimes G, G, s, t)$ with $s\left(g, g^{\prime}\right)=g^{\prime}, t\left(g, g^{\prime}\right)=g g^{\prime}$, $g, g^{\prime} \in G$, corresponding to the crossed module of groups $\left(G, G, \operatorname{id}_{G}\right)$. There is a group homomorphism $\epsilon: G \rightarrow G \rtimes G, g \mapsto(g, 1)$, satisfying $s \epsilon=1$ and $t \epsilon=\mathrm{id}_{G}$. Next, we need to consider the cat ${ }^{1}$-algebra $(\mathbb{K}(G \rtimes G) / X, \mathbb{K}(G), \overline{\mathbb{K}}(s), \overline{\mathbb{K}}(t))$, where $X=\operatorname{Ker} \mathbb{K}(s) \operatorname{Ker} \mathbb{K}(t)+\operatorname{Ker} \mathbb{K}(t) \operatorname{Ker} \mathbb{K}(s)$. Let $\pi: \mathbb{K}(G \rtimes G) \rightarrow \mathbb{K}(G \rtimes G) / X$ denote the canonical epimorphism, then we have the equalities $\overline{\mathbb{K}}(s) \pi \mathbb{K}(\epsilon)=$ $\mathbb{K}(s) \mathbb{K}(\epsilon)=0$ and $\overline{\mathbb{K}}(t) \pi \mathbb{K}(\epsilon)=\mathbb{K}(t) \mathbb{K}(\epsilon)=\mathrm{id}_{G}$. It follows that the homomorphism $\pi \mathbb{K}(\epsilon)$ takes values in $\operatorname{Ker} \overline{\mathbb{K}}(s)$ and it is a right inverse for the homomorphism

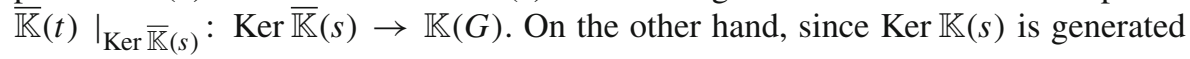
by all elements of the form $(g, 1), g \in G$, it is obvious that $\left.\pi \mathbb{K}(\epsilon) \overline{\mathbb{K}}(t)\right|_{\operatorname{Ker} \overline{\mathbb{K}}(s)}=$ $\operatorname{id}_{\operatorname{Ker} \overline{\mathbb{K}}(s)}$.

Now it is easy to see that the pair $\left(\left.\overline{\mathbb{K}}(t)\right|_{\operatorname{Ker} \overline{\mathbb{K}}(s)}, \operatorname{id}_{\mathbb{K}(G)}\right)$ is an isomorphism of crossed modules of algebras $\left(\operatorname{Ker} \overline{\mathbb{K}}(s), \mathbb{K}(G),\left.\overline{\mathbb{K}}(t)\right|_{\operatorname{Ker}} \overline{\mathbb{K}}(s)\right)$ and $(\mathbb{K}(G), \mathbb{K}(G)$, $\left.\operatorname{id}_{\mathbb{K}(G)}\right)$, which provides the required isomorphism between the functors $\mathbb{X} \mathbb{K} \circ \mathbb{E}^{\prime}{ }_{1}$ and $\mathbb{E}_{1} \circ \mathbb{K}$.

\subsection{Main results}

The following result is a natural generalization of the well-known classical adjunction between the categories Gr and UAlg.

Theorem 4.5 (cf. [10]) The functor $\mathbb{X} \mathbb{K}$ is left adjoint to the functor $\mathbb{X} \mathbb{U}$.

Proof We have to construct a natural bijection

$$
\begin{aligned}
& \operatorname{Hom}_{\mathrm{XGr}}((H, G, \mu), \mathbb{X} \mathbb{U}(R, A, \rho)) \cong \operatorname{Hom}_{\mathrm{XUAlg}}(\mathbb{X} \mathbb{K}(H, G, \mu),(R, A, \rho)), \\
& \text { for any }(H, G, \mu) \in \mathrm{XGr} \text { and }(R, A, \rho) \in \mathrm{XUAlg} \text {. }
\end{aligned}
$$


Given a morphism $(\alpha, \beta) \in \operatorname{Hom}_{\mathrm{XGr}}((H, G, \mu), \mathbb{X} \mathbb{U}(R, A, \rho))$, consider the corresponding morphism of cat ${ }^{1}$-groups

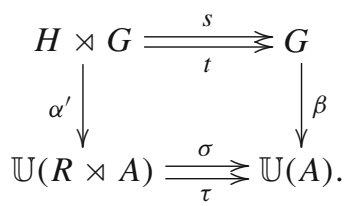

Since the functor $\mathbb{K}$ is left adjoint to the functor $\mathbb{U}$, we have the induced commutative diagram of algebras

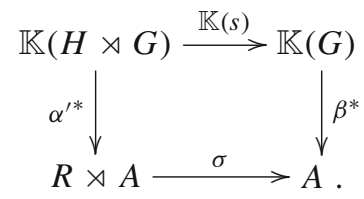

The similar diagram holds with $\mathbb{K}(s)$ replaced by $\mathbb{K}(t)$ and $\sigma$ replaced by $\tau$. Since $\operatorname{Ker} \sigma \operatorname{Ker} \tau+\operatorname{Ker} \tau \operatorname{Ker} \sigma=0$, we have uniquely defined morphism of cat ${ }^{1}$-algebras

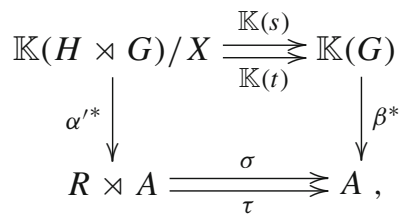

which corresponds to a uniquely defined morphism from $\operatorname{Hom}_{\mathrm{XUAlg}}(\mathbb{X} \mathbb{K}(H, G, \mu)$, $(R, A, \rho))$. The inverse assignment is similar.

Theorem 4.6 Let $(H, G, \mu)$ be a crossed module of groups. Then the category of $(H, G, \mu)$-modules over $\mathbf{k}$ is isomorphic to the category of (left) $\mathbb{X} \mathbb{K}(H, G, \mu)$ modules.

Proof By using Lemma 4.3 and Theorem 4.5, $(H, G, \mu)$-module structures on a k-homomorphism $\partial: M \rightarrow P$ are in one-to-one correspondence with $\mathbb{X} \mathbb{K}(H, G, \mu)$ module structures on it. Due to the equations (8) and (9), it is easy to check that this correspondence is functorial.

\section{References}

1. Baues, H.-J., Minian, E.G.: Crossed extensions of algebras and Hochschild cohomology. Homol. Homotopy Appl. 4(2), 63-82 (2002)

2. Beck, J.: Triples, algebras and cohomology. Ph.D. Thesis, Columbia University, 1967. Repr. Theory Appl. Categ., vol. 2, pp. 1-59 (2003)

3. Brown, R.: Groupoids and crossed objects in algebraic topology. Homol. Homotopy Appl. 1(1), 1-78 (1999) 
4. Brown, R., Higgins, P.J., Sivera, R.: Nonabelian algebraic topology. Filtered spaces, crossed complexes, cubical homotopy groupoids, EMS Tracts in Mathematics 15, European Mathematical Society (EMS), Zürich (2011)

5. Dedecker, P., Lue, A.S.-T.: A nonabelian two-dimensional cohomology for associative algebras. Bull. Am. Math. Soc. 72(6), 1044-1050 (1966)

6. Donadze, G., Inassaridze, N., Khmaladze, E., Ladra, M.: Cyclic homologies of crossed modules of algebras. J. Noncommut. Geom. 6, 749-771 (2012)

7. Elgueta, J.: Representation theory of 2-groups on Kapranov and Voevodsky's 2-vector spaces. Adv. Math. 213, 53-92 (2007)

8. Ellis, G.J.: Higher dimensional crossed modules of algebras. J. Pure Appl. Algebra. 52, 277-282 (1988)

9. Fernández Vilaboa, J.M., López López, M. P., Villanueva Novoa, E.: Cat ${ }^{1}$-Hopf algebras and crossed modules. Comm. Algebra. 35, 181-191 (2007)

10. Forrester-Barker, M.: Representations of crossed modules and cat ${ }^{1}$-groups. Ph.D. Thesis, University of Bangor, 2003. http://www.maths.bangor.ac.uk/research/ftp/theses/forrester-barker.pdf

11. Frégier, Y., Wagemann, F.: On Hopf 2-algebras. Int. Math. Res. Not. IMRN. 2011, 3471-3501 (2011)

12. Inassaridze, H., Khmaladze, E.: Hopf formulas for equivariant integral homology of groups. Proc. Amer. Math. Soc. 138(9), 3037-3046 (2010)

13. Loday, J.-L.: Spaces with finitely many non-trivial homotopy groups. J. Pure Appl. Algebra. 24, 179202 (1982)

14. Lue, A.S.-T.: Non-abelian cohomology of associative algebras. Quart. J. Math. Oxford Ser. 19(2), 159-180 (1968)

15. Norrie, K.: Actions and automorphisms of crossed modules. Bull. Soc. Math. France. 118, 129-146 (1990)

16. Paoli, S.: On the non-balanced property of the category of crossed modules in groups. J. Pure Appl. Algebra. 197, 19-22 (2005)

17. Whitehead, J.H.C.: Combinatorial homotopy II. Bull. Am. Math. Soc. 55, 453-496 (1949) 